\title{
Neurofunctional Reward Processing Changes in Cocaine Dependence During Recovery
}

\author{
Iris M Balodis', Hedy Kober', Patrick D Worhunsky', Michael C Stevens², Godfrey D Pearlson ${ }^{1,2,3}$, \\ Kathleen M Carroll' and Marc N Potenza*,1,3,4 \\ 'Department of Psychiatry, Yale University School of Medicine, New Haven, CT, USA; ${ }^{2}$ Institute of Living/Hartford Hospital and Olin \\ Neuropsychiatry Research Center, Hartford, CT, USA; ${ }^{3}$ Department of Neurobiology, Yale University School of Medicine, New Haven, CT, USA; \\ ${ }^{4}$ Child Study Center, Yale University School of Medicine, New Haven, CT, USA
}

\begin{abstract}
Although reward processing appears altered in addiction, few studies track neurofunctional changes following treatment or relate these to measures of reduced drug use. The current study examined neurofunctional alterations in reward processing in cocaine dependence (CD) pretreatment and posttreatment to determine whether these changes relate to clinically meaningul outcome indicators. Treatmentseeking CD outpatients $(N=29)$ underwent functional magnetic resonance imaging while performing a monetary incentive delay task (MIDT) pretreatment and posttreatment. The MIDT parses anticipatory from outcome phases of reward/loss processing. Abstinence indicators (negative urines, days abstinent from cocaine during follow-up) were collected throughout treatment and up to I year later. Healthy control $(\mathrm{HC})$ participants $(N=28)$ were also scanned twice with the MIDT. Relative to pretreatment, at posttreatment $C D$ participants demonstrated increased anticipatory reward activity in the midbrain, thalamus, and precuneus ( $p_{\text {FWE }}<0.05$ ). Increased midbrain activity correlated with cocaine abstinence during the I-year follow-up. Ventral striatal (VS) activity during loss anticipation correlated negatively with negative urine screens. HC group test-retest results showed decreased ventromedial prefrontal cortex activity during winning outcomes. CD-HC group-by-time differences revealed increased left inferior frontal gyrus activity in the CD group during anticipatory phases at posttreatment. In CD participants, increased posttreatment activity in dopamine-innervated regions suggests lowered thresholds in anticipatory signaling for non-drug rewards. Midbrain and VS responses may represent biomarkers associated with CD abstinence. Abstinence-related neurobiological changes occur in similar regions implicated during active use and may possibly be used to track progress during short- and long-term recovery.

Neuropsychopharmacology (2016) 41, 2112-2121; doi:10.1038/npp.2016.11; published online 17 February 2016
\end{abstract}

\section{INTRODUCTION}

Relapse rates for cocaine dependence $(\mathrm{CD})$ remain among the highest of all illicit drugs (Heyman, 2013; Vocci, 2007). Although many neuroimaging studies examine chronic effects of cocaine on cognitive processes, relatively few examine brain changes occurring with discontinued drug use (Garavan et al, 2013; Hanlon et al, 2013). Investigating neurofunctional changes associated with abstinence may provide important insights regarding strategies for cessation and relapse prevention (Garavan et al, 2013; Hanlon et al, 2013).

To date, most neuroimaging studies exploring abstinence in $\mathrm{CD}$ have been cross-sectional, often with small samples and with varied testing times (eg, posttreatment, long-term abstinence). One of the few longitudinal neuroimaging $\mathrm{CD}$

*Correspondence: Professor MN Potenza, Department of Psychiatry and Child Study Center, Yale University School of Medicine, Connecticut Mental Health Center, Room S- 104, 34 Park Street, New Haven, CT 06519, USA, Tel: +I 203974 7356, Fax: +I 203974 7366, E-mail: marc.potenza@yale.edu

Received 10 August 2015; revised I December 2015; accepted 21 December 2015; accepted article preview online 21 January 2016 studies found that, at follow-up, CD individuals demonstrated an enhanced midbrain response during a cognitivecontrol task, which inversely related to simulated cocaine choice (Moeller et al, 2012). Although limited by a small sample and the absence of a control group, this study nonetheless provides some evidence of network recovery with sustained abstinence. Greater midbrain activation has also been observed in a reward processing task comparing former, relative to current, cocaine users (Patel et al, 2013); abstinence in the former users correlated with right ventral striatum (VS) activity during anticipatory processing (Patel et al, 2013). This study is limited in its cross-sectional design; current and former cocaine users differed in their self-reported cocaine use, with length of abstinence difficult to assess in the former cocaine-using group.

The current study aimed to provide insight into the neural basis of recovery; few studies track neural changes within individuals from baseline to treatment follow-up and relate these to abstinence indicators. This study employed a longitudinal design to follow a large group of $\mathrm{CD}$ individuals and examine brain changes in generalized reward processing 
Table I Participant's Demographic Information

\begin{tabular}{lcc}
\hline & CD & HC \\
\hline$n$ & 29 & 28 \\
Male/female & $21 / 8$ & $14 / 14$ \\
Age (SD)* & $41.34(6.7)$ & $31.25(10.1)$ \\
White/Black/Hispanic/Asian/multiracial & $12 / 13 / 2 / 0 / 2$ & $18 / 9 / 1 / 1 / 0$ \\
Years of education* & $12.52(1.3)$ & $15.36(2.1)$ \\
Previous 28 days cocaine use & $15.52(6.9)$ & - \\
Previous 28 days cigarette use & $21.07(12.0)$ & - \\
Previous 28 days alcohol use & $6.45(7.7)$ & - \\
Previous 28 days marijuana use & $1.21(2.8)$ & - \\
Years cocaine use & $9.41(6.1)$ & - \\
Age at first cocaine use & $22.28(7.9)$ & - \\
Days in treatment & $53.59(31.9)$ & - \\
Sessions attended & $6.21(4.3)$ & \\
\hline * & & -
\end{tabular}

before and after treatment. Specifically, our main aims were to:

(1) Use a longitudinal, rather than cross-sectional, design to examine brain changes occurring from pretreatment to posttreatment in a large group of individuals participating in a randomized clinical trial of CD treatments. Few studies track neural changes within individuals from baseline to follow-up. Many addiction theories emphasize changes in the mesocorticolimbic dopamine system to motivationally salient stimuli across the addiction cycle; for example, increased release to drug-related cues (Goldstein et al, 2009) but diminished response to natural/generalized rewards (Volkow et al, 2007). If chronic drug use modifies dopamine signaling, then protracted abstinence may also alter activity in these regions. Consistent with previous $\mathrm{CD}$ studies (Moeller et al, 2012; Patel et al, 2013), we hypothesized that, at posttreatment, relative to pretreatment, $C D$ individuals would demonstrate functional enhancement of dopaminergically innervated brain regions, including the midbrain, thalamus, and precuneus.

(2) Examine generalized reward processing changes, particularly anticipatory processing. Many addiction treatments focus on reducing drug use by increasing the salience of non-drug-related stimuli (Prendergast et al, 2006; Vocci, 2007). Learning-based addiction theories underscore anticipatory processes as critical for associative learning mechanisms leading to habit formation (Everitt and Robbins, 2013). We hypothesized that functional enhancement of dopaminergically-innervated brain areas may occur specifically during anticipatory processing.

(3) Assess the degree to which functional changes in anticipatory processing relate to abstinence measures over time. Anticipatory processing recruits the VS, which effectively codes for reward-predicting cues, including drug cues (Knutson et al, 2001). Several cross-sectional studies link striatal reward processing with abstinence in $C D$, yet with somewhat differing results (Bustamante et al, 2013; Jia et al, 2011; Patel et al, 2013); this may relate to clinical differences, such as treatment-seeking status, length of abstinence, and recent cocaine use (Balodis and Potenza,
2015). Changes in the VS may act as a possible biomarker of relapse risk, although no study has directly examined this possibility. Given the importance of the VS in drug and reward processing, this area was selected as a region of interest (ROI) to examine relationships with abstinence indicators. Abstinence often occurs incrementally, rather than as a dichotomous process (Konova et al, 2013); negative urine screens are an objective measure of abstinence that has been identified as a clinically meaningful outcome indicator (Carroll et al, 2014). We hypothesized that pretreatmentposttreatment VS anticipatory changes would relate to negative urine screens.

(4) Examine practice effects on a reward processing task. In order to control for potential practice effects on the task, a control group was included who completed the task twice. Given the role of fronto-striatal circuitry in mediating reward, we hypothesized reduced activity in this network upon repeated testing.

\section{MATERIALS AND METHODS}

\section{Participants}

Participants consisted of 57 individuals providing written informed consent (see Table 1 and Supplementary Data for sample characteristics and diagnostic procedures). CD participants were scanned prior to treatment and at posttreatment. The average time between scanning sessions (ie, Scan1 and Scan2) for participants was 129 days $(\mathrm{SD}=66.90)$ with no significant difference between $\mathrm{CD}$ and HC groups $(F(1,55)=0.61, p>0.05)$. CD participants were treatment-seeking individuals recruited from a larger clinical research trial. CD participants received cognitive behavioral therapy with random assignment to 12 weeks of adjunct therapy and a 1-year follow-up; details of the RCT and outcomes are described in the main trial report (Carroll et al, 2016), here we focus on neural functioning and abstinence.

\section{Group Assessments}

Rather than one single measure, multiple outcome indicators have been identified as clinically meaningful (Carroll et al, 2014); here we present negative urine screens as our primary abstinence indicator. Negative urine screens comprise an objective measure of abstinence that has been identified in a recent review as a clinically meaningful indicator of treatment outcome (Carroll et al, 2014). Urine toxicology screens were obtained three times weekly during treatment and at each follow-up interview.

\section{Reward Task}

Participants completed two MIDT runs during each scanning session. Each trial includes anticipatory phases A1 (prospect of reward) and A2 (anticipation of reward receipt) and an outcome phase (OC). The task description can be found in Supplementary Data and previous publication (Patel et al, 2013).

Functional images were preprocessed using SPM5 (Welcome Functional Imaging Laboratory, London, UK), normalized to the Montreal Neurological Institute template and smoothed with a 6-mm FWHM kernel. First-level modeling 
used robust regression to reduce outlier influences (Wager et al, 2005). Motion and high-pass filter parameters comprised additional regressors of no interest. NeuroElf analysis package (www.neuroelf.net) was used for second-level random-effects analysis. Recommended cluster-extent-based thresholding analytic and reporting practices for fMRI were applied (Woo et al, 2014); correction for multiple comparisons was conducted using Monte-Carlo simulation (ie, AlphaSim), using a combined voxel-wise $(p<0.001)$ and cluster thresholds to result in a family-wise error (FWE) rate of $p_{\mathrm{FWE}}<0.05$. To investigate brain activation over time, we contrasted: (1) anticipation of monetary gain during Scan2 vs Scan1 for $\mathrm{A} 1$ and A2 phases (A1Win and A2Win, respectively); (2) anticipation of monetary loss during Scan2 vs anticipation of monetary loss during Scan 1 for A1 and A2 phases (A1Loss and A2Loss, respectively); (3) outcome win processing during Scan2 from outcome win processing during Scan1; and (4) outcome loss processing during Scan2 from outcome loss processing during Scan1. Our analyses focused on Scan2 relative to Scan1 differences within the $\mathrm{CD}$ group, and further present test-retest differences in an HC group. Additionally, between-group comparisons among the treatment-seeking $\mathrm{CD}$ group and the $\mathrm{HC}$ group were examined.

\section{ROI Analysis}

Given the small VS volume, together with evidence implicating this area in anticipatory processing, the MIDT, and $\mathrm{CD}$ pathophysiology, the VS constituted an a priori ROI specifically during this reward phase (ie, A2). The ROI on the right side was defined and localized based on rewardprocessing findings in a previous cross-sectional MIDT study in former CD users (Patel et al, 2013), reporting a relationship between right anticipatory VS activity and abstinence duration. Additionally, this lateralized region was further identified in a meta-analysis of brain responses to cocaine cues, with the right VS also linked to drug craving (Kuhn and Gallinat, 2011). Activity from a spherical ROI ( $5 \mathrm{~mm}$ radius around 12,12, -9; Figure 2) was extracted for each CD participant to examine the mean blood oxygen level-dependent percentage of signal change from baseline. Subsequently, Spearman's rho correlations in SPSS, version 17.0 (SPSS, Chicago, Illinois) tested the relationship between anticipatory VS changes and negative urine screens. Specifically, VS ROI activity during win and loss anticipation (Scan2 win cues $>$ Scan 1 win cues; Scan2 loss cues $>$ Scan 1 loss cues) during A2 was correlated with negative urine toxicology screens and Bonferroni-corrected for multiple comparisons.

\section{RESULTS}

\section{Main Effect of Time/Treatment on Anticipatory Processing in the CD Group}

No significant Scan2-Scan1 differences were observed during $\mathrm{A} 1$ phases, the A2Loss phase, or OC phases in $\mathrm{CD}$ participants.

During the A2Win phase (associated with the anticipation of reward), Scan2-Scan1 differences recruited bilateral thalamus extending to right caudate and lentiform nucleus (Table 2a; Figure 1b); precuneus; posterior cingulate extending to culmen (Figure 1a and c); and right midbrain/substantia nigra extending to lentiform nucleus (Figure 1d). A previous study in CD inversely linked enhanced midbrain response in an overlapping region with simulated cocaine choice (Moeller et al, 2012). In an effort to replicate and extend this aforementioned report, we conducted an exploratory analysis between the midbrain cluster and long-term abstinence; midbrain activity correlated with days of cocaine abstinence during follow-up $(r=0.48, p<0.01$; Figure 1e).

Changes in anticipatory VS activity and negative urines. Spearman's rho statistic between A2W posttreatment $>$ pretreatment changes and negative urine screens was not significant. The total negative urines during treatment, however, correlated inversely with A2L posttreatment $>$ pretreatment VS changes $\left(r_{s}=-0.42, p=0.05\right.$; Bonferronicorrected; Figure 2).

\section{HC Test-Retest Differences}

Within this group, significant Scan2-Scan1 differences occurred only during the OCWin phase; showing a significant decrease in ventromedial prefrontal cortex (vmPFC) extending to anterior cingulate from Scan1 to Scan2 (Table 2b, Figure $3 a$ and $b$ ).

\section{Differences Between CD Posttreatment-Pretreatment and HC Test-Retest}

There were no between-group differences during A1 or OC phases when comparing Scan2-Scan1 activity. During both A2Win and A2Loss phases, group differences appeared in left IFG driven by increased posttreatment-pretreatment activity in the CD group (Figure 3d). Groups also differed in the right superior temporal gyrus, where the $\mathrm{CD}$ group demonstrated Scan2-Scan1 increases (Table 2c; Figure 3c). Extracted signals from these regions covarying for age did not alter results.

\section{DISCUSSION}

Using a longitudinal design, the current study examined reward-processing changes in $\mathrm{CD}$ participants during treatment. Significant neurofunctional changes occurred predominantly during anticipatory processing with additional relationships to a clinically meaningful outcome indicator. Inclusion of $\mathrm{HC}$ subjects permitted characterizing test-retest effects and $\mathrm{CD}-\mathrm{HC}$ group differences.

\section{Within-Group CD Differences}

Increased anticipatory reward processing. In line with our hypothesis, changes in anticipatory processing occurred from baseline to the end of treatment in the CD group. Specifically, CD participants showed increased anticipatory reward activity in the midbrain and thalamus areas extending to striatal regions. Our findings replicate and extend findings from two prior CD studies. Our longitudinal findings of increased midbrain activity from baseline to the end of treatment are consistent with prior cross-sectional CD data indicating greater midbrain activity in the former, 
Table 2 Scan2-Scan I Differences During the MIDT

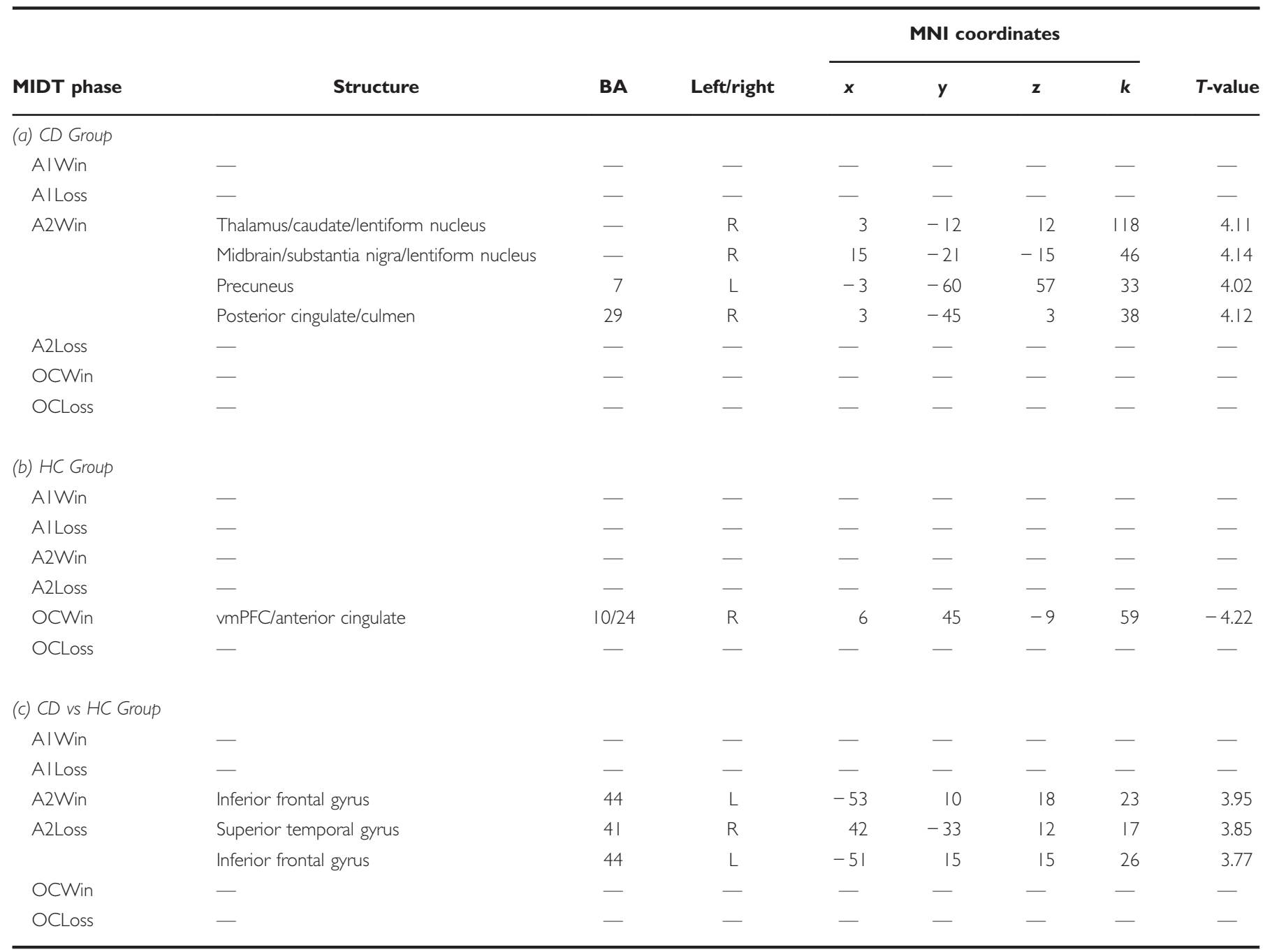

Abbreviations: AILoss, prospect of loss phase; A2Loss, anticipation of loss; AIWin, prospect of reward phase; A2Win, anticipation of reward; BA, Brodman's area; k, cluster; MIDT, monetary incentive delay task; OCLoss, notification of loss; OCWin, notification of reward; vmPFC, ventromedial prefrontal cortex.

relative to current, cocaine users using the same rewardprocessing task (Patel et al, 2013). Additionally, our findings are consistent with other longitudinal data in CD participants performing a cognitive-control task with a pretreatment-posttreatment design (Moeller et al, 2012). Notably, the midbrain cluster coordinates reported here during anticipatory reward show significant overlap with those reported previously in which midbrain activity change correlated negatively with explicit cocaine selections on a neuropsychological drug-choice task (Moeller et al, 2012). Here, in an exploratory analysis, we extend this finding to link midbrain activity with real-world abstinence using 1-year follow-up data (Figure 1e). Notably, this correlation with the A2Win phase, one reflecting anticipatory activity with motor preparatory effects removed, suggests increased midbrain recruitment during passive anticipation of a rewarding non-drug cue. Nonetheless, the prior and present studies administered two different tasks (drug Stroop and MIDT, respectively) assessing distinct cognitive domains; enhanced midbrain response may therefore represent a biomarker for approach motivation relating to choice behavior and longer-term cocaine use outcomes in $\mathrm{CD}$. Altogether, these findings suggest the possibility that midbrain-related activation improvement with abstinence could relate to increases in attentional effort occurring with drug discontinuation (Sarter et al, 2006). Nevertheless, increased attention may itself represent a form of cognitive incentive, driven either through explicit and/or implicit motivational forces that might increase with recovery (Sarter et al, 2006). Future studies might disentangle motivational from attentional or working-memory processes occurring with recovery.

The midbrain contains dopaminergic cell bodies with ascending projections to striatal and thalamic regions responsive to reward-predicting cues (Schultz et al, 1997). Although BOLD fMRI cannot measure neurotransmitter activity directly, greater anticipatory activity in this network posttreatment may indicate a recovery of dopamine-related activity and endogenous response to non-drug rewards (Choi et al, 2006). Some evidence links dopamine D2 receptor availability with monetary expectation response in $\mathrm{CD}$ (Asensio et al, 2010), suggesting that resting availability of 


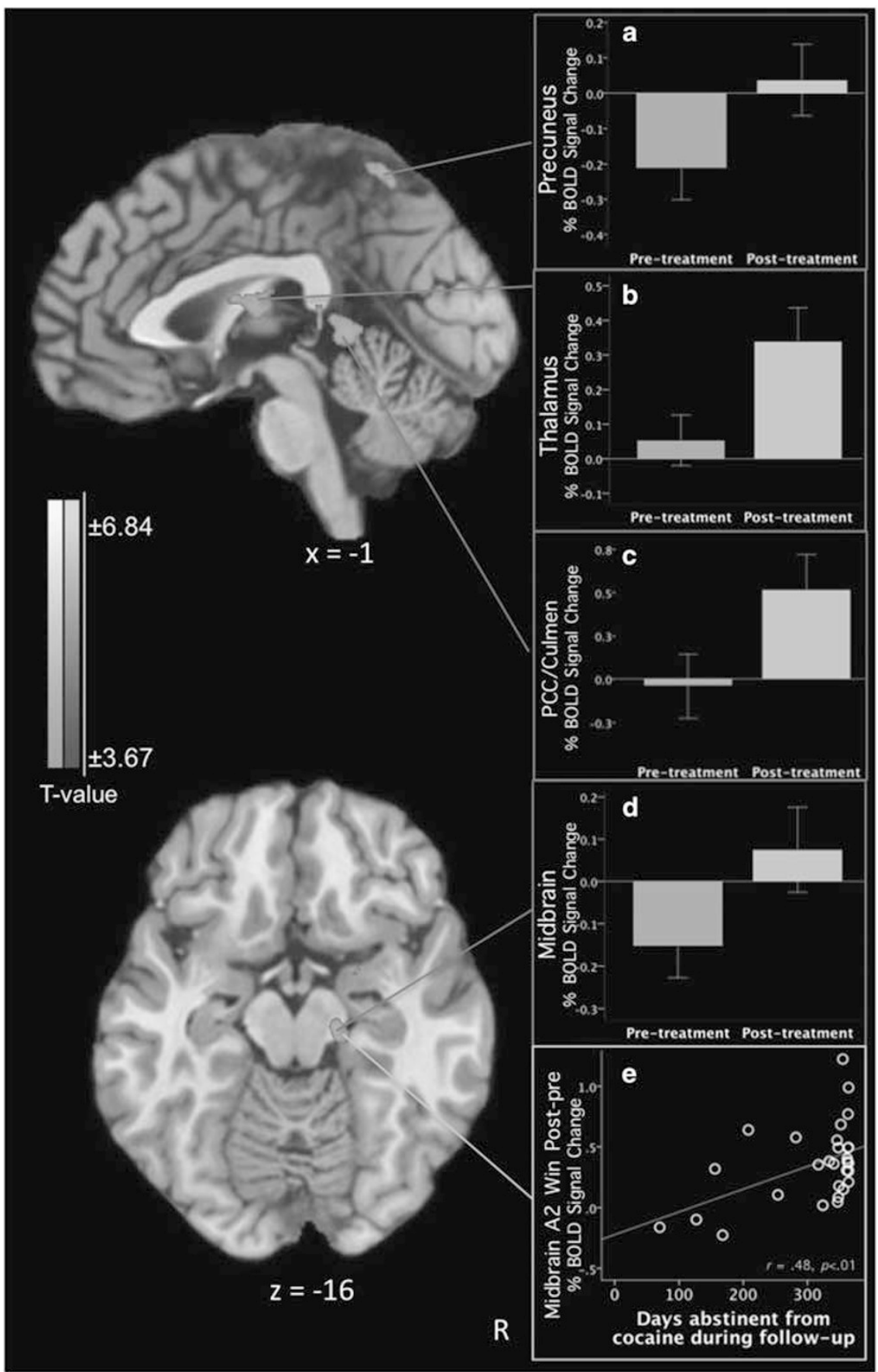

Figure I Posttreatment-pretreatment differences on the Monetary Incentive Delay Task in the cocaine-dependent (CD) group ( $n=29)$ during the A2 winning phase (A2W, associated with the anticipation of potentially winning money). (a) The percentage of blood-oxygen-level-dependent (BOLD) signal change in the precuneus cluster during pretreatment and posttreatment; (b) BOLD signal change in the thalamus cluster during pretreatment and posttreatment; (c) BOLD signal change in the posterior cingulate (PCC)/culmen cluster during pretreatment and posttreatment; (d) BOLD signal change in the midbrain cluster during pretreatment and posttreatment; (e) scatterplot demonstrating a positive correlation between the midbrain A2W cluster difference and days abstinent from cocaine during follow-up $(r=0.48, p<0.0$ I). All contrast maps are thresholded at an uncorrected level of $p<0.00$ I two-tailed and family-wise-error-corrected at $p<0.05$. Blue color demonstrates areas where subjects show relatively less activation at posttreatment vs pretreatment, and red color indicates where participants show relatively greater activation at posttreatment vs pretreatment. The right side of the brain is on the right. A full color version of this figure is available at the Neuropsychopharmacology journal online.

this receptor may predict functional responses to monetary reinforcers. Additionally, these findings provide some index of abstinence duration necessary to observe brain activation changes; increases in dopamine-innervated regions (eg, midbrain, striatum) occurred following 4 months. Although the midbrain is susceptible to respiratory artifacts during imaging (Raj et al, 2001), the pattern of pretreatmentposttreatment activation increases through dopaminer- 


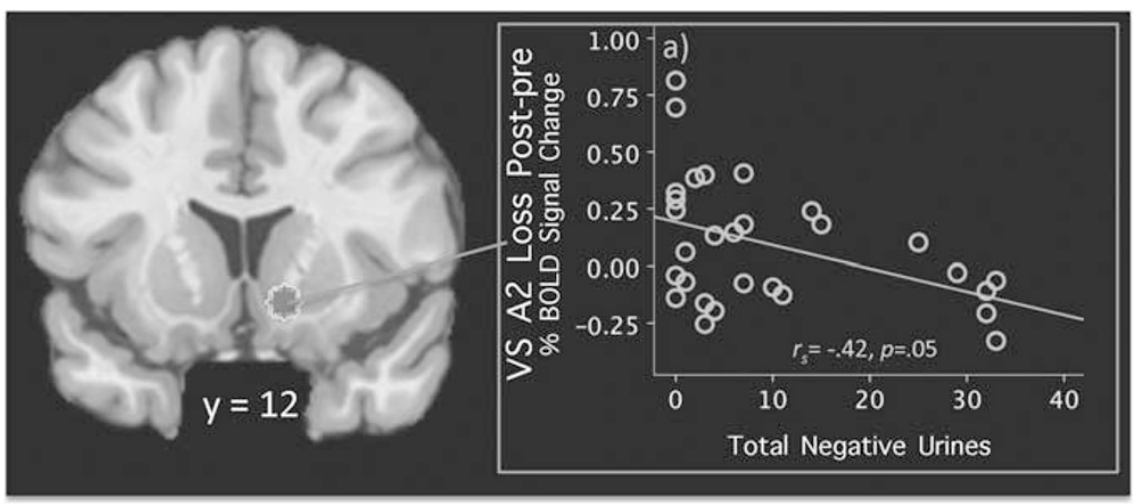

Figure 2 Coronal view of the ventral striatal Region of Interest (ROI) using coordinates reported by Patel et al (20 I 3). A blue spot indicates a 5-mm sphere around the ventral striatum (VS) on the right $(12,12,-9)$ side. Scatterplots depict the percentage of blood-oxygen-level-dependent (BOLD) signal change extracted from the 5-mm ROI during the A2L Loss phase (posttreatment > pretreatment) correlated with (a) the total negative urines during treatment period $\left(r_{s}=-0.42, p=0.05\right)$. A full color version of this figure is available at the Neuropsychopharmacology journal online.

gically-innervated regions, together with the striking overlap in cluster coordinates with a prior pretreatment-posttreatment fMRI study in CD, lend support to this as a biologically significant effect. Consistent with learning theories of addiction underscoring the importance of associative learning mechanisms, our findings highlight that recovery processes may recruit the same reward networks affected by chronic drug use. Furthermore, the plasticity of responsiveness in this circuitry support the goal of many therapeutic interventions in addiction aiming to increase the salience of non-drug-related cues. Future studies can focus on relating these recovery patterns with specific therapies and subjective effects in individuals.

Relationship between functional changes in VS anticipatory processing and abstinence. The VS ROI analyses link negative incentive signaling with individual variation in reduction of cocaine use within treatment as measured by negative urine screens. Our findings relating VS activity changes with abstinence are consistent with the right VS as a core region responding to cocaine cues and drug craving (Kuhn and Gallinat, 2011). Preclinical studies show cravingrelated VS dopamine signaling instigating drug-seeking behavior (Saunders et al, 2013); similarly, our findings link negative incentive VS signaling with cocaine. Notably, links with cocaine use measures occurred during loss anticipation, highlighting the possible role of negative incentive signaling in recovery.

Although increased VS signaling might be expected with reduction of cocaine use, the negative correlation between VS changes and negative urines is nonetheless consistent with findings from two cross-sectional CD MIDT studies linking reduced VS activity with greater abstinence (Bustamante et al, 2013; Patel et al, 2013). Our VS results extend these findings, demonstrating not only reduced VS activity, but lower VS fluctuations over time relate to stable remission. These findings provide insight into mixed findings in the literature, as cross-sectional designs may capture volatility in VS signaling in current users. These findings hint at dynamic VS dysregulation, particularly to anticipatory cues, as an important clinical index in $\mathrm{CD}$, potentially related to neurotransmitter bioavailability at different phases of the abuse-abstinence-relapse cycle. For example, actively using individuals may produce a greater dynamic response when challenged, through the presence of drug metabolites (ie, recent cocaine use) synergistically affecting the reward signal or through VS neuroadaptations from recent drug use. Moreover, imbalances in tonic-phasic signaling of cells from chronic drug exposure may dynamically alter the excitatory tone of cells across distinctive recovery phases (Phillips et al, 2003); similarly, alterations in VS dopamine receptor availability and affinity are observed in $\mathrm{CD}$ and relate to fMRI responses to non-drug rewards 3 years later (Asensio et al, 2010; Volkow et al, 2007). The data presented here suggest that abstinence-related neurobiological changes occur in similar regions showing neurofunctional alterations at pretreatment and that these might be used to track progress during protracted recovery. Additionally, correlational findings linking VS recruitment with changes in cocaine use provide support for this area as a possible predictor of better outcomes over time.

\section{Test-Retest Differences in HCs}

To date, few studies have examined test-retest effects in HCs on the MIDT; therefore, investigating these changes is important in characterizing neural responses associated with repeated task exposure. Differences emerged in the OCWin phase, with a significant reduction in vmPFC activity at retest. Given the ascribed role of the vmPFC in tracking monetary reward outcomes (Knutson et al, 2003), these findings suggest diminished outcome-associated reward responses and possibly reduced integration of incentive information with repeated MIDT exposure. These findings were observed in the absence of affective differences to winning outcomes across the two scans.

\section{Differences Between CD and HC}

Few prospective studies have examined brain-based mechanisms of change; to our knowledge, this is the first CD study 
using fMRI at multiple time points to compare CD-HC differences in reward processing over time. Group differences emerged in the left IFG exclusively during the A2 phases, whereby CD participants demonstrated significant increases relative to HC participants, who showed decreased activity here. The IFG is implicated in various functions, including
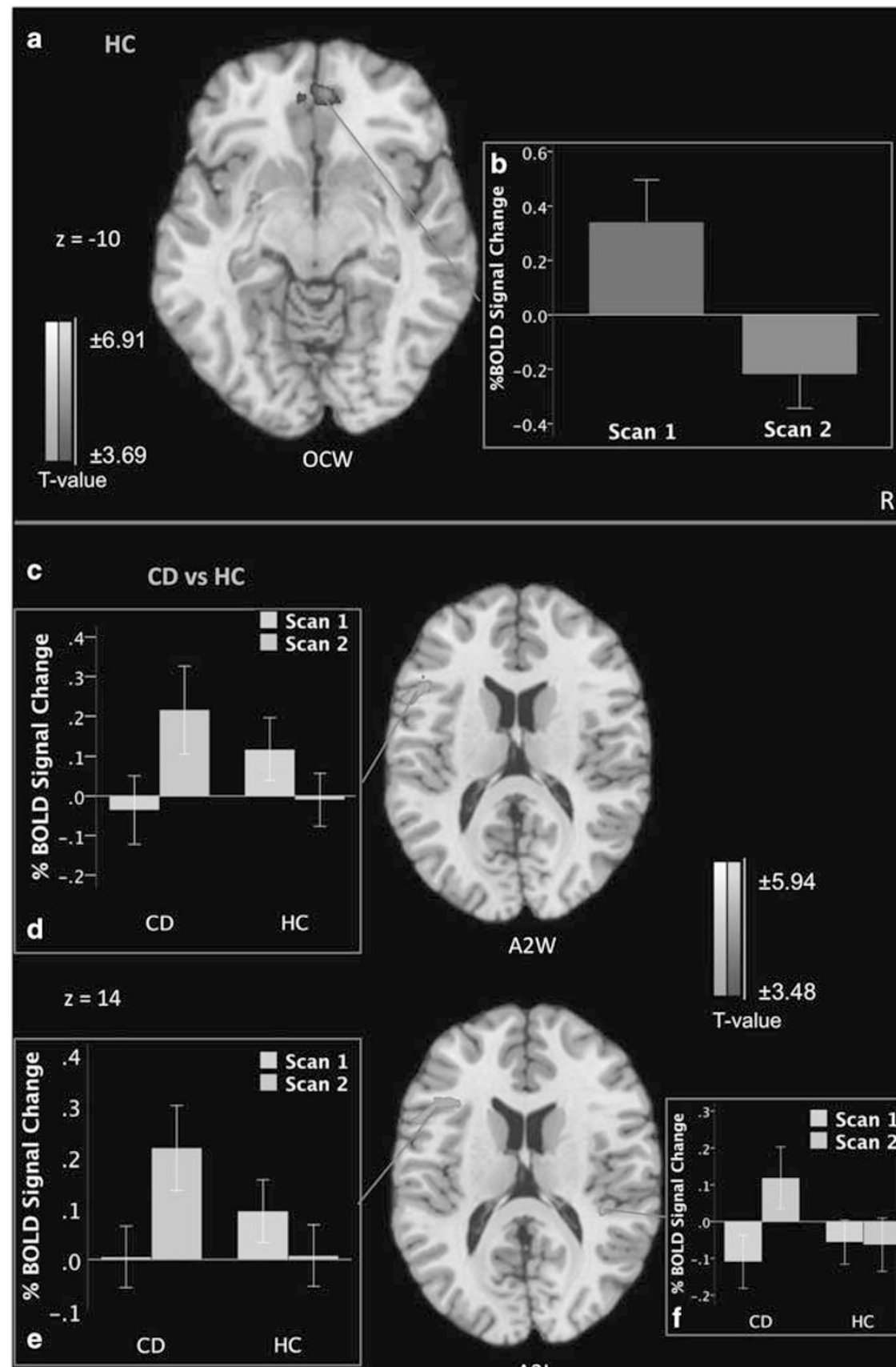

$\mathrm{A} 2 \mathrm{~L}$

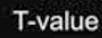

value

Figure 3 Scan2-Scan I differences on the Monetary Incentive Delay Task in (a) the HC group ( $n=28)$ during the outcome winning phase (OCW, associated with the receipt of reward); (b) depicts the percentage of blood-oxygen-level-dependent (BOLD) signal change in the ventromedial prefrontal cortex (vmPFC; $z=-10$ ) in the HC group for their first and second scans. (c) Scan2-Scanl contrast between the CD group ( $n=29)$ and the HC group $(n=28)$; (d) depicts the percentage of blood-oxygen-level-dependent (BOLD) signal change in the left inferior frontal gyrus $(z=\mid 4)$ in the $C D$ and $H C$ groups for their first and second scans during the A2 winning phase (A2W, associated with the anticipation of potentially winning money); (e) depicts the percentage of blood-oxygen-level-dependent (BOLD) signal change in the left inferior frontal gyrus $(z=\mid 4)$ in the CD and HC groups for their first and second scans during the $\mathrm{A} 2$ losing phase (A2L, associated with the anticipation of potentially losing money); (e) depicts the percentage of blood-oxygen-level-dependent (BOLD) signal change in the right superior temporal gyrus $(z=14)$ in the $C D$ and $H C$ groups for their first and second scans during the $A 2$ losing phase. All contrast maps are thresholded at an uncorrected level of $p<0.00$ I two-tailed and family-wise-error-corrected at $p<0.05$. Blue color demonstrates areas where subjects show relatively less activation in the indicated contrast map, and red color indicates where participants show relatively greater activation. The right side of the brain is on the right. A full color version of this figure is available at the Neuropsychopharmacology journal online. 
attention and behavior monitoring in goal-directed behavior (Corbetta and Shulman, 2002), and is described as a hub for limbic-executive functions (Bari and Robbins, 2013). Rather than subcortical areas, these findings characterize neurofunctional test-retest differences between $\mathrm{CD}$ and $\mathrm{HC}$ individuals as occurring during anticipatory processing in cortical areas involved in attention and executive control. Continued longitudinal fMRI investigations during recovery will establish whether normalization of functioning occurs between groups or whether dynamic processes persist with sustained abstinence. Comparisons with $\mathrm{HC}$ populations are critical for additionally tracking normative neuroadaptive changes occurring over time and further gauging neurobiological recovery profiles in addiction.

\section{Strengths, Limitations, and Future Directions}

The current study is novel in demonstrating changes in reward processing occurring in a CD population following interventions and linking these with clinically meaningful abstinence measures. This study also benefits from a comparatively large sample, a within-subject longitudinal design, rigorous fMRI analytical thresholds to reduce the rate of false positives, and further includes hypothesis-driven ROIs informed by the research literature. We report changes in similar/overlapping brain regions with comparable measures as reported by other groups (Bustamante et al, 2013; Moeller et al, 2012; Patel et al, 2013) and in populations with considerably more abstinence, thereby contributing to replication and broader generalizations beyond the current study. Therefore, results from the current study are not only consistent with previous CD study findings but also novel in presenting recovery-related findings; to our knowledge, this is the first study to longitudinally examine generalized reward processing changes in a $\mathrm{CD}$ population. Our prospective design using a well-validated reward-processing task contributes to the field's understanding of anticipatory processing fluctuations in addiction. These findings are particularly meaningful in the context of addiction literature, given the significant role of incentive signaling to rewardpredicting cues thought to underlie addictive processes. Specifically, our findings of increased reward anticipation in mesocorticolimbic circuitry over time provide some evidence for recovery of generalized reward processing in this population. These findings are distinct from those of Moeller et al (2012), one of the few other studies also using a pretreatment-posttreatment design in a CD population. Moeller et al (2012) employed a drug Stroop task, which assesses cognitive domains of attention and inhibition specific to drug cue, rather than generalized reward processing. Our findings are also unique from Patel et al (2013), who employed a cross-sectional design and focused on loss outcome processing on the MIDT.

The inclusion of an HC group further permits examination of test-retest changes occurring with the MIDT and comparisons with the CD group. The current study examined outcomes across time in individuals participating in a range of treatments; it was not possible to evaluate treatment-specific effects or ascribe changes in neural activity to the effects of reduced cocaine use or specific effects of treatment. Additionally, a recent report demonstrates an inverse relationship between depressive symptoms and right VS activity on the MIDT (Hagele et al, 2015). In the current study, depression scores were not collected in the $\mathrm{CD}$ group. Nonetheless, excluding the seven participants with a lifetime history of depression did not alter the correlations between $\mathrm{A} 2 \mathrm{~L}$ and outcome measures and, in fact, slightly strengthened them.

Gaining a better idea of neurofunctional changes during treatment represents an important first step in understanding the neurobiology of successful abstinence (Garavan et al, 2013; Hanlon et al, 2013). Additionally, our findings of increased precuneus/PCC activity during A2Win are consistent with a recent meta-analysis citing common effects of pharmacological and cognitive-based interventions in these areas (Konova et al, 2013). Finally, while the MIDT version administered dissociates specific phases of reward processing, BOLD fMRI cannot directly gauge neurotransmitter systems underlying changes in the motivational salience signals. Nonetheless, the results have implications for narrowing in on particular mechanisms and therapeutic targets. More research will be important in clarifying whether incentive motivational signals to non-drug cues directly reflect an adapted/ recovered capacity to recruit dopamine synthesis. Further, studying VS signal volatility and adaptations over time merits additional investigations and could advance understanding of recovery processes.

\section{CONCLUSIONS}

This controlled, prospective study demonstrates a functional enhancement of dopaminergically innervated brain regions occurring specifically during anticipatory processing in a CD group following treatment. An exploratory analysis further linked midbrain activity with abstinence 1 year later. Additionally, functional changes in the VS relate to an objective measure of abstinence (urine screens). Altogether, this is one of the first longitudinal studies demonstrating how incentive signaling reflects recovery processes in CD. These findings suggest that both midbrain and VS responses during anticipatory processing may represent biomarkers for approach motivation relating to abstinence in CD. Although previous studies demonstrate striatal dopamine surges following drug-cue exposure in $\mathrm{CD}$ that positively correlate with craving measures (Boileau et al, 2007; Volkow et al, 2006; Wong et al, 2006), here we show that individual changes in non-drug anticipatory responsivity in the striatum relate to abstinence. The current study demonstrates abstinence-related neurofunctional changes during recovery, particularly relating to incentive salience signal readjustments linked to measures of reduced cocaine use over time. These findings have clinical relevance, as the effectiveness of many therapeutic interventions relies on the dynamic response to rewarding non-drug cues (Vocci, 2007). These findings support the idea that over time and with less expensive approaches to imaging, neural data outcome might be applied in the future to more specifically evaluate the clinical impact of particular therapies. Future studies longitudinally investigating individuals at multiple months and even years after treatment could clarify which changes are long-lasting and most predictive of sustained recovery. 


\section{FUNDING AND DISCLOSURE}

Dr Potenza has received financial support or compensation for the following: he has consulted for Ironwood, Lundbeck, Shire, INSYS and RiverMend Health; has received research support from Mohegan Sun Casino, the National Center for Responsible Gaming, and Pfizer Pharmaceuticals; has participated in surveys, mailings or telephone consultations related to drug addiction, impulse control disorders, or other health topics; has consulted for law offices and gambling entities on issues related to impulse control disorders; provides clinical care in the Connecticut Department of Mental Health and Addiction Services Problem Gambling Services Program; has performed grant reviews for the National Institutes of Health and other agencies; has guestedited journal sections; has given academic lectures in grand rounds, CME events, and other clinical or scientific venues; and has generated books or book chapters for publishers of mental health texts. The other authors declare no conflict of interest.

\section{ACKNOWLEDGMENTS}

This study was supported by the National Institute on Drug Abuse grants R01 DA019078, P50 DA09241, P20 DA027844, R01 DA035058, R01 DA020908, and K12 DA00167.

\section{REFERENCES}

Asensio S, Romero MJ, Romero FJ, Wong C, Alia-Klein N, Tomasi D et al (2010). Striatal dopamine D2 receptor availability predicts the thalamic and medial prefrontal responses to reward in cocaine abusers three years later. Synapse 64: 397-402.

Balodis IM, Potenza MN (2015). Anticipatory reward processing in addicted populations: a focus on the monetary incentive delay task. Biol Psychiatry 77: 434-444.

Bari A, Robbins TW (2013). Inhibition and impulsivity: behavioral and neural basis of response control. Prog Neurobiol 108: 44-79.

Boileau I, Dagher A, Leyton M, Welfeld K, Booij L, Diksic M et al (2007). Conditioned dopamine release in humans: a positron emission tomography [11C]raclopride study with amphetamine. J Neurosci 27: 3998-4003.

Bustamante JC, Barros-Loscertales A, Costumero V, FuentesClaramonte P, Rosell-Negre P, Ventura-Campos N et al (2013). Abstinence duration modulates striatal functioning during monetary reward processing in cocaine patients. Addict Biol. 19: 885-894.

Carroll KM, Kiluk BD, Nich C, DeVito EE, Decker S, LaPaglia D et al (2014). Toward empirical identification of a clinically meaningful indicator of treatment outcome: features of candidate indicators and evaluation of sensitivity to treatment effects and relationship to one year follow up cocaine use outcomes. Drug Alcohol Depend 137: 3-19.

Carroll KM, Nich C, Petry NM, Eagan DA, Shi JM, Ball SA et al (2016). A randomized factorial trial of disulfiram and contingency management to enhance cognitive behavioral therapy for cocaine dependence. Drug Alcohol Depend pii: S0376-8716(16)00008-9. doi:10.1016/j.drugalcdep.2015.12.036 (e-pub ahead of print).

Choi JK, Chen YI, Hamel E, Jenkins BG (2006). Brain hemodynamic changes mediated by dopamine receptors: role of the cerebral microvasculature in dopamine-mediated neurovascular coupling. Neuroimage 30: 700-712.
Corbetta M, Shulman GL (2002). Control of goal-directed and stimulus-driven attention in the brain. Nat Rev Neurosci 3: 201-215.

Everitt BJ, Robbins TW (2013). From the ventral to the dorsal striatum: devolving views of their roles in drug addiction. Neurosci Biobehav Rev 37: 1946-1954.

Garavan H, Brennan KL, Hester R, Whelan R (2013). The neurobiology of successful abstinence. Curr Opin Neurobiol 23: 668-674.

Goldstein RZ, Tomasi D, Alia-Klein N, Honorio Carrillo J, Maloney T, Woicik PA et al (2009). Dopaminergic response to drug words in cocaine addiction. J Neurosci 29: 6001-6006.

Hagele C, Schlagenhauf F, Rapp M, Sterzer P, Beck A, Bermpohl F et al (2015). Dimensional psychiatry: reward dysfunction and depressive mood across psychiatric disorders. Psychopharmacology (Berl) 232: 331-341.

Hanlon CA, Beveridge TJ, Porrino LJ (2013). Recovering from cocaine: insights from clinical and preclinical investigations. Neurosci Biobehav Rev 37: 2037-2046.

Heyman GM (2013). Quitting drugs: quantitative and qualitative features. Annu Rev Clin Psychol 9: 29-59.

Jia Z, Worhunsky PD, Carroll KM, Rounsaville BJ, Stevens MC, Pearlson GD et al (2011). An initial study of neural responses to monetary incentives as related to treatment outcome in cocaine dependence. Biol Psychiatry 70: 553-560.

Knutson B, Adams CM, Fong GW, Hommer D (2001). Anticipation of increasing monetary reward selectively recruits nucleus accumbens. J Neurosci 21: RC159.

Knutson B, Fong GW, Bennett SM, Adams CM, Hommer D (2003). A region of mesial prefrontal cortex tracks monetarily rewarding outcomes: characterization with rapid event-related fMRI. Neuroimage 18: 263-272.

Konova AB, Moeller SJ, Goldstein RZ (2013). Common and distinct neural targets of treatment: changing brain function in substance addiction. Neurosci Biobehav Rev 37: 2806-2817.

Kuhn S, Gallinat J (2011). Common biology of craving across legal and illegal drugs - a quantitative meta-analysis of cue-reactivity brain response. Eur J Neurosci 33: 1318-1326.

Moeller SJ, Tomasi D, Woicik PA, Maloney T, Alia-Klein N, Honorio J et al (2012). Enhanced midbrain response at 6-month follow-up in cocaine addiction, association with reduced drugrelated choice. Addict Biol 17: 1013-1025.

Patel KT, Stevens MC, Meda SA, Muska C, Thomas AD, Potenza MN et al (2013). Robust changes in reward circuitry during reward loss in current and former cocaine users during performance of a monetary incentive delay task. Biol Psychiatry 74: 529-537.

Phillips PE, Stuber GD, Heien ML, Wightman RM, Carelli RM (2003). Subsecond dopamine release promotes cocaine seeking. Nature 422: 614-618.

Prendergast M, Podus D, Finney J, Greenwell L, Roll J (2006). Contingency management for treatment of substance use disorders: a meta-analysis. Addiction 101: 1546-1560.

Raj D, Anderson AW, Gore JC (2001). Respiratory effects in human functional magnetic resonance imaging due to bulk susceptibility changes. Phys Med Biol 46: 3331-3340.

Sarter M, Gehring WJ, Kozak R (2006). More attention must be paid: the neurobiology of attentional effort. Brain Res Rev 51: 145-160.

Saunders BT, Yager LM, Robinson TE (2013). Cue-evoked cocaine "craving": role of dopamine in the accumbens core. J Neurosci 33: 13989-14000.

Schultz W, Dayan P, Montague PR (1997). A neural substrate of prediction and reward. Science 275: 1593-1599.

Vocci FJ (2007). Can replacement therapy work in the treatment of cocaine dependence? And what are we replacing anyway? Addiction 102: 1888-1889. 
Volkow ND, Fowler JS, Wang GJ, Swanson JM, Telang F (2007). Dopamine in drug abuse and addiction: results of imaging studies and treatment implications. Arch Neurol 64: 1575-1579.

Volkow ND, Wang GJ, Telang F, Fowler JS, Logan J, Childress AR et al (2006). Cocaine cues and dopamine in dorsal striatum: mechanism of craving in cocaine addiction. J Neurosci 26: 6583-6588.

Volkow ND, Wang GJ, Telang F, Fowler JS, Logan J, Jayne M et al (2007). Profound decreases in dopamine release in striatum in detoxified alcoholics: possible orbitofrontal involvement. J Neurosci 27: 12700-12706.
Wager TD, Keller MC, Lacey SC, Jonides J (2005). Increased sensitivity in neuroimaging analyses using robust regression. Neuroimage 26: 99-113.

Wong DF, Kuwabara H, Schretlen DJ, Bonson KR, Zhou Y, Nandi A et al (2006). Increased occupancy of dopamine receptors in human striatum during cue-elicited cocaine craving. Neuropsychopharmacology 31: 2716-2727.

Woo CW, Krishnan A, Wager TD (2014). Cluster-extent based thresholding in fMRI analyses: pitfalls and recommendations. Neuroimage 91: 412-419. 